\title{
AN APPROXIMATE MATRIX INVERSION PROCEDURE BY PARALLELIZATION OF THE SHERMAN-MORRISON FORMULA
}

\author{
KENTARO MORIYA ${ }^{凶 1}$, LINJIE ZHANG $^{2}$ and TAKASHI NODERA ${ }^{3}$
}

(Received 11 February, 2009; revised 5 November, 2009)

\begin{abstract}
The Sherman-Morrison formula is one scheme for computing the approximate inverse preconditioner of a large linear system of equations. However, parallelizing a preconditioning approach is not straightforward as it is necessary to include a sequential process in the matrix factorization. In this paper, we propose a formula that improves the performance of the Sherman-Morrison preconditioner by partially parallelizing the matrix factorization. This study shows that our parallel technique implemented on a PC cluster system of eight processing elements significantly reduces the computational time for the matrix factorization compared with the time taken by a single processor. Our study has also verified that the Sherman-Morrison preconditioner performs better than ILU or MR preconditioners.
\end{abstract}

\section{Introduction}

In this study we consider the solution of large and sparse linear systems of equations

$$
A \boldsymbol{x}=\boldsymbol{b},
$$

where the coefficient matrix $A$ is of the order of $n \times n$ and the solution vector $\boldsymbol{x}$ and right-hand side $\boldsymbol{b}$ are $n \times 1$ vectors. Since iterative methods often require a large number of iterations to solve a linear system of equations (1.1), it is usually necessary to use some kind of a preconditioning technique. Recently, Bru et al. [1] proposed computing a preconditioner based on the Sherman-Morrison formula, and showed that it consistently performed well. However, it is not an easy task to parallelize the computation of the preconditioner in such a formula, since the $k$ th column vector depends on the first $k-1$ column vectors in the matrix factorization.

\footnotetext{
${ }^{1}$ Head Office, Nikon System Inc., Japan; e-mail: kmoriya @ nikon-sys.co.jp.

${ }^{2}$ College of Mathematical Sciences, Ocean University of China, Japan; e-mail: zhanglinjie@ hotmail.com.

${ }^{3}$ Department of Mathematics, Faculty of Science and Technology, Keio University, Japan; e-mail: nodera@math.keio.ac.jp.

(C) Australian Mathematical Society 2010, Serial-fee code 1446-1811/2010 \$16.00
} 
In this study, we propose a technique that partially parallelizes the computation of this preconditioner. Two test problems demonstrate that the parallel performance of our technique implemented on a PC cluster system is significantly faster than the solution of the problems by the use of a single processor. We also demonstrate that our technique can accelerate the convergence of the residual norm.

\section{The Sherman-Morrison preconditioner}

In the recurrence equation,

$$
A_{k}=A_{k-1}+\boldsymbol{p}_{k} \boldsymbol{q}_{k}^{\mathrm{T}},
$$

where $A_{k}$ and $A_{k-1}$ are nonsingular matrices and $\boldsymbol{p}_{k}, \boldsymbol{q}_{k}$ are nonzero vectors. When the Sherman-Morrison formula is applied to Equation (2.1),

$$
A_{k}^{-1}=A_{k-1}^{-1}-r_{k}^{-1} A_{k-1}^{-1} \boldsymbol{p}_{k} \boldsymbol{q}_{k}^{\mathrm{T}} A_{k-1}^{-1},
$$

where

$$
r_{k}=1+\boldsymbol{q}_{k}^{\mathrm{T}} A_{k-1}^{-1} \boldsymbol{p}_{k} .
$$

Summing Equation (2.2) from $k=1$ to $k=n$ gives

$$
A^{-1}=A_{0}^{-1}-\sum_{k=1}^{n} r_{k}^{-1} A_{k-1}^{-1} \boldsymbol{p}_{k} \boldsymbol{q}_{k}^{\mathrm{T}} A_{k-1}^{-1},
$$

where $A^{-1}=A_{n}^{-1}$. Equation (2.4) can be expressed as

$$
A_{0}^{-1}-A^{-1}=\Psi \Omega^{-1} \Xi^{\mathrm{T}}
$$

where

$$
\Psi=\left[A_{0}^{-1} \boldsymbol{p}_{1}, A_{1}^{-1} \boldsymbol{p}_{2}, \ldots, A_{n-1}^{-1} \boldsymbol{p}_{n},\right], \quad \Omega^{-1}=\operatorname{diag}\left[r_{1}^{-1}, r_{2}^{-1}, \ldots, r_{n}^{-1}\right]
$$

and

$$
\Xi=\left[\boldsymbol{q}_{1}^{\mathrm{T}} A_{0}^{-1}, \boldsymbol{q}_{2}^{\mathrm{T}} A_{1}^{-1}, \ldots, \boldsymbol{q}_{n}^{\mathrm{T}} A_{n-1}^{-1}\right]
$$

Define

$$
\boldsymbol{u}_{k}:=\boldsymbol{p}_{k}-\sum_{i=1}^{k-1} \frac{\boldsymbol{v}_{i}^{\mathrm{T}} A_{0}^{-1} \boldsymbol{p}_{k}}{r_{i}} \boldsymbol{u}_{i}, \quad \boldsymbol{v}_{k}:=\boldsymbol{q}_{k}-\sum_{i=1}^{k-1} \frac{\boldsymbol{q}_{k}^{\mathrm{T}} A_{0}^{-1} \boldsymbol{u}_{i}}{r_{i}} \boldsymbol{v}_{i}
$$

so that $A_{k-1}^{-1} \boldsymbol{p}_{k}=A_{0}^{-1} \boldsymbol{u}_{k}$ and $\boldsymbol{q}_{k}^{\mathrm{T}} A_{k-1}^{-1}=\boldsymbol{v}_{k}^{\mathrm{T}} A_{0}^{-1}$ are satisfied. Substituting these two relations into Equations (2.3) and (2.5),

$$
r_{k}=1+\boldsymbol{q}_{k}^{\mathrm{T}} A_{0}^{-1} \boldsymbol{u}_{k}
$$

and

$$
A_{0}^{-1}-A^{-1}=A_{0}^{-1} U \Omega^{-1} V^{\mathrm{T}} A_{0}^{-1},
$$

respectively, where $U=\left(\boldsymbol{u}_{1}, \boldsymbol{u}_{2}, \ldots, \boldsymbol{u}_{n}\right)$ and $V=\left(\boldsymbol{v}_{1}, \boldsymbol{v}_{2}, \ldots, \boldsymbol{v}_{n}\right)$. Note that $A_{0}$, $\boldsymbol{p}_{k}$ and $\boldsymbol{q}_{k}$ are still included in the relations (2.6), and these values have to be 
determined. The matrix $A_{0}^{-1}-A^{-1}$ should approximate the inverse of $A$. According to Bru et al. [1], the simplest method for achieving this approximation is to take

$$
A_{0}=s I, \quad \boldsymbol{p}_{k}=\boldsymbol{e}_{k}, \quad \boldsymbol{q}_{k}=\left(\boldsymbol{a}^{k}-s \boldsymbol{e}_{k}\right)^{\mathrm{T}},
$$

where $\boldsymbol{a}^{k}$ and $\boldsymbol{e}_{k}$ are the $k$ th column vectors of $A$ and the $k$ th entry of the identity vector is 1.0, respectively. Bru et al. [1] demonstrated that $s=1.5 \times\|A\|_{\infty}$ is a suitable choice for this scalar in (2.9). If we adopt this selection approach (2.9), Equations (2.6) and (2.7) are transformed to

$$
\begin{gathered}
\boldsymbol{u}_{k}=\boldsymbol{p}_{k}-\sum_{i=1}^{k-1} \frac{\left(v_{i}\right)_{k}}{s r_{i}} \boldsymbol{u}_{i}, \\
\boldsymbol{v}_{k}=\boldsymbol{q}_{k}-\sum_{i=1}^{k-1} \frac{\boldsymbol{q}_{k}^{\mathrm{T}} \boldsymbol{u}_{i}}{s r_{i}} \boldsymbol{v}_{i}, \\
r_{k}=1+\frac{\left(\boldsymbol{v}_{k}\right)_{k}}{s},
\end{gathered}
$$

where $\left(\boldsymbol{v}_{k}\right)_{k}$ is the $k$ th entry of $\boldsymbol{v}_{k}$. If we set the left-hand side of Equation (2.8) to be equal to the preconditioner $M^{-1}$, the formula for computing the preconditioner is then

$$
M^{-1}=s^{-1} I-A^{-1}=s^{-2} U \Omega^{-1} V^{\mathrm{T}} .
$$

Since $M^{-1}$ is the product of $U, \Omega^{-1}$ and $V^{\mathrm{T}}$, it can be computed by using Equations (2.10)-(2.12). From Equation (2.13) it is clear that $M^{-1}$ differs from $A^{-1}$ only in its diagonal entries. To reduce computational costs, not all of the nonzero entries of $U$ and $V$ are computed; significantly small entries are usually neglected.

\section{Parallel implementation}

3.1. Parallel matrix decomposition The drawback of computing $M^{-1}$ using Equation (2.13) is that the computation of $\boldsymbol{u}_{k}$ and $\boldsymbol{v}_{k}$ where $k=2, \ldots, n$ is dependent on the vectors $\boldsymbol{u}_{1}, \ldots, \boldsymbol{u}_{k-1}, \boldsymbol{v}_{1}, \ldots, \boldsymbol{v}_{k-1}$, and the scalars $r_{1}, \ldots, r_{k-1}$. The parallelization of these computations is therefore not straightforward and so we propose a parallel technique that computes Equations (2.10) and (2.11).

3.2. Allocation of $\boldsymbol{u}_{\boldsymbol{k}}, \boldsymbol{v}_{\boldsymbol{k}}$ and $\boldsymbol{r}_{\boldsymbol{k}}$ to PEs Assuming that the orders of matrices $U$, $\Omega$ and $V$ are $n$ and the number of processing elements (PEs) is $d$, the $(m l+1)$ th to $(l+1) m$ th column vectors of $U, V$ and diagonal entries of $\Omega^{-1}$ are allocated to the $l$ th PE, where $m$ is the number of column vectors and diagonal entries are covered by one PE. In the case of the matrix $U$, for instance, the allocation is given by

$$
U=\{\underbrace{\boldsymbol{u}_{1}, \ldots, \boldsymbol{u}_{m}}_{\mathrm{PE}_{0}}, \underbrace{\boldsymbol{u}_{m+1}, \ldots, \boldsymbol{u}_{2 m}}_{\mathrm{PE}_{1}}, \ldots, \underbrace{\boldsymbol{u}_{n-m+1}, \ldots, \boldsymbol{u}_{n}}_{\mathrm{PE}_{d-1}}\},
$$

where $\mathrm{PE}_{l}$ is the $l$ th $\mathrm{PE}$ for $l=0, \ldots, d-1$.

An additional assumption we have made is that $n$ can be divided by $d$, with $m=n / d$. Note, however, that we can also proceed when $n$ is not divisible by $d$. This 
is solved through an allocation where the zeroth to $(\tilde{d}-1)$ th PEs cover $\lfloor n / d\rfloor+1$, and the $\tilde{d}$ th to $(d-1)$ th PEs cover $\lfloor n / d\rfloor$ column vectors and diagonal entries, where $\tilde{d}=n \bmod d$ and $\lfloor n / d\rfloor$ is the integer part of $n / d$.

3.3. Parallel implementation of the preconditioner From Equations (2.10) and (2.11) we know that $\boldsymbol{u}_{k}$ and $\boldsymbol{v}_{k}$ depend on $\boldsymbol{u}_{1}, \ldots, \boldsymbol{u}_{k-1}, \boldsymbol{v}_{1}, \ldots, \boldsymbol{v}_{k-1}$ and $r_{1}, \ldots, r_{k-1}$. Owing to this dependency, under normal circumstances the $l$ th PE is unable to start computing these vectors until the zeroth to $(l-1)$ th PEs have finished computing. However, the computation of Equations (2.10) and (2.11) can be partially parallelized. These two equations can be separated into three terms:

$$
\begin{array}{ll}
\boldsymbol{u}_{k}=\boldsymbol{p}_{k}-\sum_{i=1}^{l m} \frac{\left(v_{i}\right)_{k}}{s r_{i}} \boldsymbol{u}_{i}-\sum_{i=l m+1}^{k-1} \frac{\left(v_{i}\right)_{k}}{s r_{i}} \boldsymbol{u}_{i}, \quad \forall k=l m+1, \ldots,(l+1) m, \\
\boldsymbol{v}_{k}=\boldsymbol{q}_{k}-\sum_{i=1}^{l m} \frac{\boldsymbol{q}_{k}^{\mathrm{T}} \boldsymbol{u}_{i}}{s r_{i}} \boldsymbol{v}_{i}-\sum_{i=l m+1}^{k-1} \frac{\boldsymbol{q}_{k}^{\mathrm{T}} \boldsymbol{u}_{i}}{s r_{i}} \boldsymbol{v}_{i}, \quad \forall k=l m+1, \ldots,(l+1) m .
\end{array}
$$

The second term of the right-hand sides of Equations (3.1) and (3.2) cannot be computed until values are received from the other PEs, but the third term can be computed without values from any other PE. The problem is that the computation of the third term cannot be started until all of the vectors included in the second term have been received. If $\mathrm{PE}_{0}$ transfers $\boldsymbol{u}_{1}, \ldots, \boldsymbol{u}_{m}$ and $\boldsymbol{v}_{1}, \ldots, \boldsymbol{v}_{m}$ to the other PEs after computing all of these vectors, it cannot work with the other PEs simultaneously. However, if $\mathrm{PE}_{0}$ computes only $\tilde{m}(<m)$ column vectors, it can work with the other $\mathrm{PEs}$ in parallel after transferring these vectors, since $\mathrm{PE}_{0}$ still has the remaining column vectors to compute. During the first step, while $\mathrm{PE}_{0}$ is computing the first to $\tilde{m}$ th column vectors, the other PEs are idle.

During the second step, however, $\mathrm{PE}_{0}$ computes the $(\tilde{m}+1)$ th to $2 \tilde{m}$ th column vectors and transfers them while the other PEs update their covering column vectors $\boldsymbol{u}_{k}$ and $\boldsymbol{v}_{k}$ using the column vectors $\boldsymbol{u}_{1}, \ldots, \boldsymbol{u}_{\tilde{m}}$ and $\boldsymbol{v}_{1}, \ldots, \boldsymbol{v}_{\tilde{m}}$, received from $\mathrm{PE}_{0}$ during the first step. During step three, while $\mathrm{PE}_{0}$ is computing the $(2 \tilde{m}+1)$ th to $3 \tilde{m}$ th columns, the other PEs are updating their covering column vectors using the column vectors $\boldsymbol{u}_{\tilde{m}+1}, \ldots, \boldsymbol{u}_{2 \tilde{m}}$ and $\boldsymbol{v}_{\tilde{m}+1}, \ldots, \boldsymbol{v}_{2 \tilde{m}}$ received from $\mathrm{PE}_{0}$ during step two. Subsequent steps follow a similar pattern. Defining $G_{k}=\left\{\boldsymbol{u}_{k}, \boldsymbol{v}_{k}, r_{k}\right\}, G_{1}, \ldots, G_{k-1}$ are required for the computation of $G_{k}$. Based on this, $G_{k}(k=1, \ldots, n)$ can be computed in parallel as shown in Figure 1.

When $\mathrm{PE}_{0}$ has finished computing its covering $G_{k}, \mathrm{PE}_{1}$ then works in the same way as $\mathrm{PE}_{0}$, and transfers its covering $G_{k}$ to the $\mathrm{PEs}$ from $\mathrm{PE}_{2}$ to $\mathrm{PE}_{d-1}$. During this stage, although $\mathrm{PE}_{0}$ is idle, the other PEs can work in parallel. Here, $\tilde{m}$ is defined as the block number, and the appropriate block number will be dependent on the specifications of the system. The scheme of parallelization is shown in Figure 2, where tolU and tolV are the thresholds. Entries of $\boldsymbol{u}_{k}$ and $\boldsymbol{v}_{k}$ lower than these thresholds are neglected. 


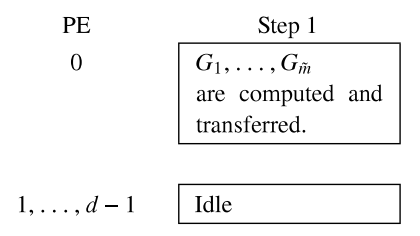

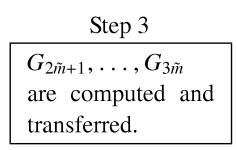

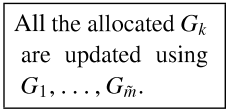



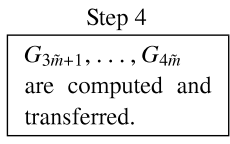

All the allocated $G_{k}$ are updated using $G_{2 \tilde{m}+1}, \ldots, G_{3 \tilde{m}}$.

FIGURE 1. A parallel scheme for computing $G_{k}$.

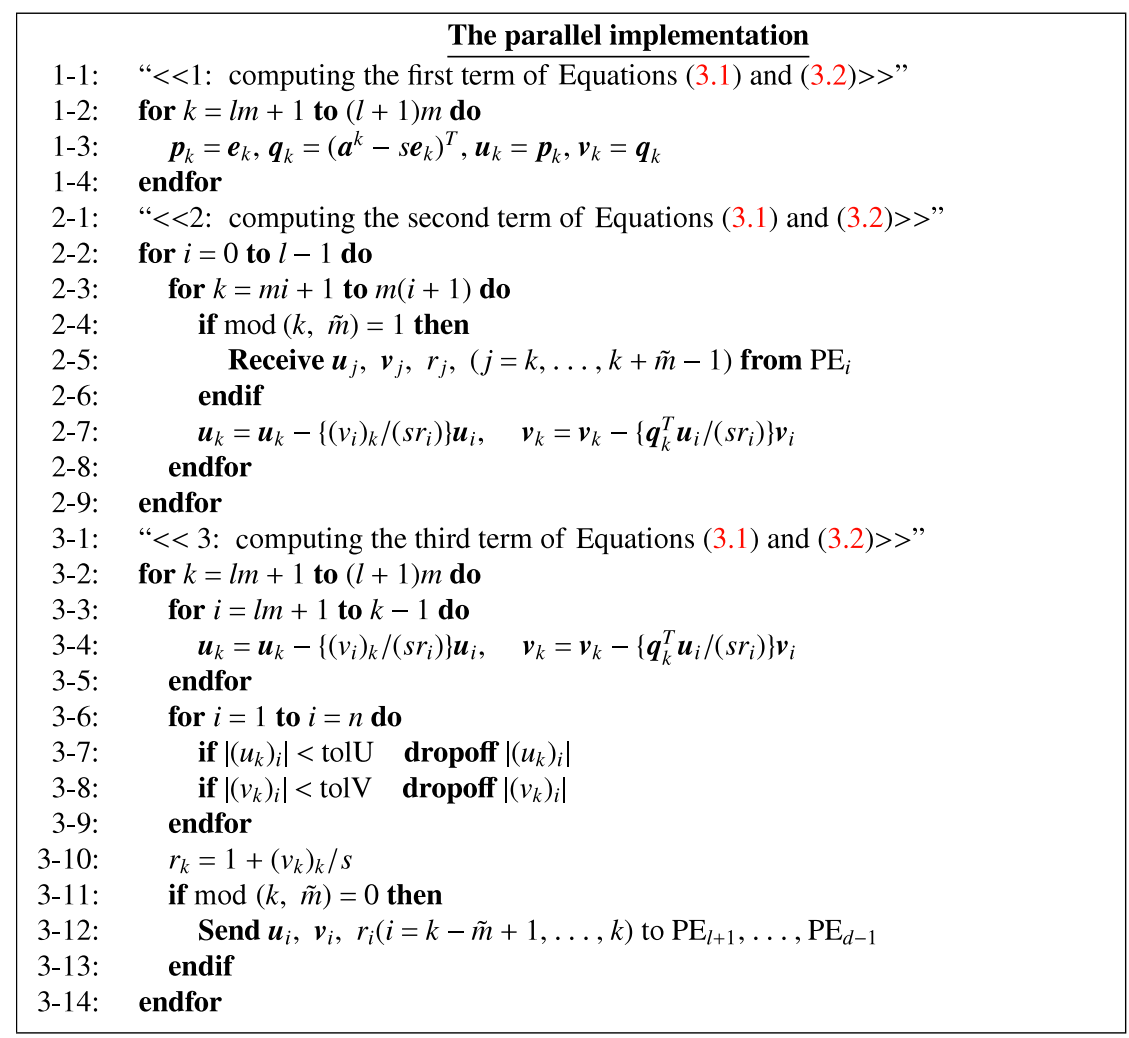

FIgURE 2. The parallel implementation of the Sherman-Morrison preconditioner for the $l$ th PE.

\section{Numerical experiments}

We used a PC cluster system with four nodes configured with the Linux Fedora Core 4 operating system. Each node was configured with two Pentium Xeon $3.6 \mathrm{GHz}$ processors and $1 \mathrm{~GB}$ memory. The total number of PEs was eight. The MPI communication library was used. The main experiments were used to analyse the parallel performance of the computation of the Sherman-Morrison preconditioner 
TABLE 1. The relationship between the number of blocks and the computation time of the ShermanMorrison preconditioner for Example 1. The column containing underlined computation times shows the optimum value of $\tilde{m}$.

\begin{tabular}{llcccccccc}
\hline & $\tilde{m}$ & 4608 & 2304 & 1152 & 576 & 288 & 144 & 72 & 36 \\
\hline \multirow{2}{*}{ time (s) } & (a) tolU, tolV $=0.1$ & 67 & 51 & 49 & 48 & 48 & 48 & 48 & $\underline{48}$ \\
& (b) tolU, tolV $=0.01$ & 471 & 365 & 325 & 318 & 317 & 317 & 318 & $\underline{312}$ \\
\hline
\end{tabular}

based on the proposed technique, and test the convergence of the preconditioned GMRES $(m)$ algorithm [3]. Regarding the convergence test, as well as the present preconditioner, we also applied the ILU factorization and the MR method [2] to the GMRES $(m)$ algorithm in order to compare the performance of the present preconditioner with these alternative preconditioners. The stopping criterion was

$$
\frac{\left\|\boldsymbol{r}_{i}\right\|_{2}}{\|\boldsymbol{b}\|_{2}}<10 \times 10^{-12}
$$

where $\boldsymbol{r}_{i}$ was the $i$ th step of the $\operatorname{GMRES}(m)$ algorithm. We parallelized the implementations of all of the preconditioners as well as the GMRES $(m)$ algorithm.

EXAMPle 1. Consider the boundary value problem described by Simoncini [4] given by the solution of a partial differential equation

$$
\begin{gathered}
-\frac{d}{d x}\left[\{\exp (-x y)\} u_{x}\right]-\frac{d}{d y}\left[\{\exp (x y)\} u_{y}\right]+10.0\left(u_{x}+u_{y}\right)-60.0 u=f(x, y), \\
\left.u(x, y)\right|_{\partial \Theta}=1+x y,
\end{gathered}
$$

in the region $\Theta=[0,1]^{2}$. We discretized this problem using a five-point difference scheme with $192^{2}$ grid points to obtain a linear system of order 36,864 . In the initial experiments we determined the computation time of the Sherman-Morrison preconditioner for this system for a selection of values of $\tilde{m}$.

The data in Table 1 shows that the computation of the Sherman-Morrison preconditioner is most efficient when $\tilde{m}=36$. Based on this, we set $\tilde{m}$ to 36 in the following experiments. We also used a different number of PEs to analyse the parallel performance of this preconditioner which was computed using this technique.

Table 2 shows that a speedup of around a factor of six or seven is possible when eight PEs are used, where $\tilde{s}=s /\left(1.5\|A\|_{\infty}\right)$ and $s=1.5\|A\|_{\infty}$ or $s=1.5\|A\|_{\infty} \times 10$. See Table 3 for the convergence test of the preconditioned $\operatorname{GMRES}(m)$ algorithm. Note that the computation time of the $\operatorname{GMRES}(m)$ algorithm includes the preconditioning time.

In Table 3, the parameters "tol" and "imax" are the thresholds of the dropoff and iteration of the MR method, respectively. When using the ILU or MR preconditioners, 
TABLE 2. The parallel performance of the computation time of the Sherman-Morrison preconditioner for Example 1.

\begin{tabular}{ccccc}
\hline & \multicolumn{4}{c}{ Number of PEs } \\
\cline { 2 - 5 }$\tilde{s}$ & 1 & 2 & 4 & 8 \\
\hline $10^{0}$ & 1.00 & 1.95 & 3.83 & 7.01 \\
$10^{1}$ & 1.00 & 1.85 & 3.45 & 6.11 \\
\hline
\end{tabular}

TABLE 3. The computation time of the iterations required to satisfy criterion (4.1) for Example 1. SM represents the Sherman-Morrison preconditioner and imax the number of iterations of the MR method. The computation time of GMRES $(m)$ algorithm includes the preconditioning time and "-" denotes cases where the residual norm could not converge within two hours.

\begin{tabular}{|c|c|c|c|c|c|c|c|}
\hline \multirow{2}{*}{\multicolumn{2}{|c|}{ Preconditioner }} & \multicolumn{6}{|c|}{ Iterative solver } \\
\hline & & \multicolumn{2}{|c|}{ GMRES(20) } & \multicolumn{2}{|c|}{ GMRES(30) } & \multicolumn{2}{|c|}{ GMRES(40) } \\
\hline Name & Time & Time & Iter & Time & Iter & Time & Iter \\
\hline $\mathrm{SM}\left(\right.$ tolU, tolV $\left.=0.1, \tilde{s}=10^{0}\right)$ & 48 & - & - & 200 & 12514 & 111 & 4453 \\
\hline $\mathrm{SM}\left(\right.$ tolU, tolV $\left.=0.1, \tilde{s}=10^{1}\right)$ & 87 & 92 & 341 & 94 & 465 & 95 & 477 \\
\hline $\mathrm{SM}\left(\right.$ tolU, tolV $\left.=0.01, \tilde{s}=10^{0}\right)$ & 315 & 346 & 1160 & 331 & 565 & 327 & 382 \\
\hline $\mathrm{SM}\left(\right.$ tolU, tolV $\left.=0.01, \tilde{s}=10^{1}\right)$ & 560 & 605 & 1075 & 578 & 425 & 578 & 396 \\
\hline $\operatorname{ILU}(0)$ & 0.24 & - & - & - & - & - & - \\
\hline ILU(1) & 0.35 & - & - & - & - & - & - \\
\hline ILU(2) & 0.48 & - & - & - & - & 28 & 934 \\
\hline $\operatorname{MR}(\operatorname{tol}=0.1, \operatorname{imax}=2)$ & 60 & - & - & - & - & - & - \\
\hline $\operatorname{MR}(\operatorname{tol}=0.1, \operatorname{imax}=1)$ & 25 & - & - & - & - & - & - \\
\hline $\operatorname{MR}(\operatorname{tol}=0.01, \operatorname{imax}=2)$ & 65 & - & - & 159 & 10616 & - & - \\
\hline $\operatorname{MR}(\operatorname{tol}=0.01$, imax $=1)$ & 29 & - & - & - & - & 87 & 6021 \\
\hline
\end{tabular}

the GMRES $(m)$ algorithm sometimes converged much faster than was the case when using the Sherman-Morrison preconditioner. However, since in most cases these two preconditioners were ineffective, their use did not lead to a converged solution. On the other hand, with only one exception, the GMRES $(m)$ algorithm with the ShermanMorrison preconditioner converged. Therefore, it seems that this preconditioner performs more robustly than the other two preconditioners.

Example 2. We studied two kinds of linear systems, coefficient matrices of which are described in Table 4 (see also [5]).

In both of these linear systems, all of the entries of the right-hand side $\boldsymbol{b}$ were 1.0. As in Example 1, we measured the appropriate $\tilde{m}$, analysed the parallel performance and compared the performance of the three kinds of preconditioners by using the $\operatorname{GMRES}(m)$ algorithm. The results are presented in Tables 5-7. 
TABLE 4. The properties of the coefficient matrices in Example 2.

\begin{tabular}{cccl}
\hline Name & Nonzeros & Order & Feature \\
\hline ecl32 & 347,097 & 51,993 & Circuit simulation \\
af23560 & 460,456 & 23,560 & Transient stability analysis of a Navier-Stokes solver \\
\hline
\end{tabular}

TABLE 5. The relationship between the number of blocks and the computation time of the ShermanMorrison preconditioner in Example 2. Columns containing an underlined computation time show the optimum value of $\tilde{m}$ for a given case.

\begin{tabular}{lrrrrrrrr}
\hline (a-1). & ecl32, tolU, tolV $=0.1$ & & & & & \\
$\tilde{m}$ & 6500 & 3250 & 1625 & 813 & 407 & 204 & 102 & 51 \\
Time (s) & 241 & 175 & $\underline{157}$ & 159 & 160 & 161 & 161 & 161 \\
(a-2). & ecl32, tolU, tolV $=0.01$ & & & & & \\
$\tilde{m}$ & 6500 & 3250 & 1625 & 813 & 407 & 204 & 102 & 51 \\
Time (s) & 1371 & 1090 & 942 & 901 & $\underline{896}$ & 902 & 911 & 913 \\
(b-1). af23560, tolU, tolV =0.1 & & & & & \\
$\tilde{m}$ & 2945 & 1473 & 737 & 369 & 185 & 93 & 47 & 24 \\
Time (s) & 1477 & 1196 & 1059 & 1007 & $\underline{989}$ & 1003 & 1027 & 1027 \\
\hline
\end{tabular}

TABLE 6. The parallel performance of the computation time of the Sherman-Morrison preconditioner in Example 2.

\begin{tabular}{ccccc}
\hline & \multicolumn{4}{c}{ Number of PEs } \\
\cline { 2 - 5 }$\tilde{s}$ & 1 & 2 & 4 & 8 \\
\hline $10^{0}$ & 1.00 & 1.67 & 2.84 & 4.45 \\
$10^{1}$ & 1.00 & 1.60 & 2.72 & 4.37 \\
\hline
\end{tabular}

Note that for the case tolU, tolV $=0.01$ for the system af 23560 , the preconditioner did not converge within two hours, and thus its results are not shown in Table 5. Table 6 shows the parallel performance of the Sherman-Morrison preconditioner when using the af 23560 system. As can be seen, computing this preconditioner using eight PEs is about 4.5 times faster than when using a single PE. Table 7 also shows the computation time and iterations for the preconditioned $\operatorname{GMRES}(m)$ algorithm. However, since this iterative solver with the ILU or MR preconditioner did not converge in every single case considered, no results are shown for these preconditioners. In contrast, the GMRES $(m)$ algorithm using the Sherman-Morrison preconditioner converged when using the af 23560 system where tolU, tolV $=0.1$, and when using the ecl32 system 
TABLE 7. The computation time of the iterations required to satisfy (4.1) for Example 2. SM denotes the Sherman-Morrison preconditioner and "_" denotes cases where the residual norm could not converge within two hours. The computation time of the $\operatorname{GMRES}(m)$ algorithm includes the preconditioning time.

\begin{tabular}{|c|c|c|c|c|c|c|c|c|}
\hline & \multirow{2}{*}{\multicolumn{2}{|c|}{ Preconditioner }} & \multicolumn{6}{|c|}{ Iterative solver } \\
\hline & & & \multicolumn{2}{|c|}{ GMRES(20) } & \multicolumn{2}{|c|}{ GMRES(30) } & \multicolumn{2}{|c|}{ GMRES(40) } \\
\hline & Name & Time & Time & Iter & Time & Iter & Time & Iter \\
\hline \multirow[t]{4}{*}{ (a) ecl32 } & $\mathrm{SM}\left(\right.$ tolU, tolV $\left.=0.1, \tilde{s}=10^{0}\right)$ & 157 & - & - & - & - & - & - \\
\hline & $\mathrm{SM}\left(\right.$ tolU, tolV $\left.=0.1, \tilde{s}=10^{1}\right)$ & 234 & - & - & - & - & - & - \\
\hline & $\mathrm{SM}\left(\right.$ tolU, tolV $\left.=0.01, \tilde{s}=10^{0}\right)$ & 956 & 1016 & 797 & 988 & 408 & 971 & 231 \\
\hline & $\mathrm{SM}\left(\right.$ tolU, tolV $\left.=0.01, \tilde{s}=10^{1}\right)$ & 1343 & 1427 & 819 & 1387 & 415 & 1365 & 221 \\
\hline \multirow[t]{2}{*}{ (b) af 23560} & $\mathrm{SM}\left(\right.$ tolU, tolV $\left.=0.1, \tilde{s}=10^{0}\right)$ & 1068 & 1161 & 759 & 1147 & 621 & 1138 & 549 \\
\hline & $\mathrm{SM}\left(\right.$ tolU, tolV $\left.=0.1, \tilde{s}=10^{1}\right)$ & 2175 & 2311 & 740 & 2285 & 649 & 2266 & 544 \\
\hline
\end{tabular}

where tolU, tolV $=0.01$. Thus, it can be seen that only this particular preconditioner is effective for the convergence of the residual norm.

\section{Conclusions}

A technique that allows the parallel implementation of the Sherman-Morrison preconditioner has been explored in this study. Application of this technique to two numerical test problems demonstrated that the computation of this preconditioner by this technique using eight PEs was 4.5-7 times faster than when using a single PE. The numerical results of the convergence test also showed that the ShermanMorrison preconditioner often performed more effectively than either the ILU or MR preconditioners.

\section{References}

[1] R. Bru, J. Credán, J. Marín and J. Mas, "Preconditioning sparse nonsymmetric linear systems with the Sherman-Morrison formula", SIAM J. Sci. Comput. 25 (2003) 701-715.

[2] M. Grote and T. Huckel, "Parallel preconditioning with sparse approximate inverses", SIAM J. Sci. Comput. 18 (1997) 838-853.

[3] Y. Saad and M. K. Schultz, "GMRES: a generalized minimal residual algorithm for solving nonsymmetric linear systems", SIAM J. Sci. Stat. Comput. 7 (1986) 856-869.

[4] Y. Simoncini, "On the convergence of restarted Krylov subspace method", SIAM J. Matrix Anal. Appl. 22 (2000) 430-452.

[5] "University of Florida sparse matrix collection", online http://www.cise.ufl.edu/research/sparse/matrices. 